\title{
Comparison of output power for solar cells with standard and structured ribbons
}

Wolfgang Muehleisen ${ }^{1, a}$, Lukas Neumaier ${ }^{1}$, Christina Hirschl $^{1}$, Thomas Maier ${ }^{2}$, Michael Schwark ${ }^{3}$, Siegfried Seufzer ${ }^{4}$, Rene Battistutti ${ }^{5}$, Mathias Pedevilla ${ }^{6}$, Jög Scheurer ${ }^{7}$, and Robert Lorenz ${ }^{8}$

${ }^{1}$ CTR Carinthian Tech Research AG, Europastr. 12, 9524 Villach/St.Magdalen, Austria

2 AIT Austrian Institute of Technology GmbH, Department of Health \& Environment, Donau-City-Straße 11210 Vienna, Austria

3 AIT Austrian Institute of Technology GmbH, Department of Energy, Giefinggasse 2, 1210 Vienna, Austria

${ }^{4}$ KIOTO Photovoltaics GmbH, Industriepark Str. 1, 9300 St.Veit/Glan, Austria

5 Energetica Energietechnik GmbH, Adi-Dassler Gasse 6, 9073 Klagenfurt-Viktring, Austria

6 Ulbrich of Austria GmbH, Industriestr. 1, 7052 Müllendorf, Austria

7 Polytec PT GmbH, Polytec-Platz 1-7, 76337 Waldbronn, Germany

8 Teamtechnik GmbH, Planckstraße 40, 71691 Freiberg a. N., Germany

Received: 2 February 2016 / Received in final form: 9 June 2016 / Accepted: 13 June 2016

(C) Muehleisen et al., published by EDP Sciences, 2016

\begin{abstract}
The optical loss due to the busbar grid and soldered interconnector ribbons on a three busbar standard multicrystalline silicon solar cell's front side is at $2.3 \%$. One way to reduce this optical loss on cell level and in a photovoltaic (PV) module is to use deep structured ribbons as cell connectors. The standard soldered, flat ribbon is replaced with a glued, multiple structured ribbon. The investigation of shiny soldered flat ribbons and multiple structured ribbons in single-cell mini modules demonstrates the light angle dependency and the benefit for the structured alternative. Additional yield measurements for conventional photovoltaic modules with soldered flat and glued multiple structured ribbons technologies were studied under laboratory conditions as well as in outdoor measurements. The simulations and the experimental findings confirmed that the new structured ribbon design increases the short circuit current and the yield by about $2 \%$.
\end{abstract}

\section{Introduction}

In general, the power output of a solar cell can be improved by reducing the amount of light reflected by the metallic busbars. Standard solar cells with a cell area of $243.4 \mathrm{~cm}^{2}$ lose $2.3 \%$ in short circuit current due to shadowing of the busbars with an entire area of $5.5 \mathrm{~cm}^{2}$. Several ideas have been tried out to compensate this loss mechanism. One idea is to paint the ribbons with white pigments for diffusing light [1]. A second attempt is to use many small round ribbons called smart wire technology (SWT) [2]. The third approach is to use the total reflection effect in combination with the glass/air interface and the structured ribbons [3-6]. These ribbons are called light harvesting (LHR) or light capturing ribbons (LCR). The effect of LCRs in comparison with flat standard ribbons is of major interest within this research.

A certain amount of light that enters the solar cell will be reflected by the cell's wiring. In a conventional cell this

\footnotetext{
a e-mail: wolfgang.muehleisen@ctr.at
}

light is lost: structured ribbon cells modify the path of the reflected light so that it is internally reflected at the glass air interface of the module and absorbed by the cell. This paper uses experiments to evaluate if this strategy increases the cell's output power, as well as simulations to understand the respective mechanism. We have found that cells with structured ribbons increase the power output by about $2 \%$ in comparison to conventional cells, making this approach economically attractive.

In a conventional three busbar solar cell, the interconnection ribbons are flat, metallic, and highly reflective and cover $2.3 \%$ of the cell's surface area. Because of their composition and geometry, light reflected by the ribbon is transmitted to the glass/air boundary and furthermore lost. If the ribbon surface is structured, light will be reflected over a wider range of angles. A specific amount of the reflected light rays will impinge on the glass/air boundary with a certain angle, resulting in total internal reflection. Subsequently, this internally reflected light will be available for energy conversion. Instead of modifying 
the composition of the ribbon's reflective surface with pigments, it is possible and in fact easier to modify its geometry.

A metallic ribbon with a triangular cross-section could potentially achieve total internal reflection for almost all of the light reflected from the ribbon. Of course this depends on the geometry, the position of the sun, the cell and the cross-section. This paper will use theory, simulations and experiments to determine the range of angles over which a structured ribbon gives a significant improvement in the short circuit current and furthermore power and yield of the module.

\section{State of the art}

\subsection{Multiple structured ribbons - benefit for PV modules}

After their invention ten years ago, nowadays structured solar interconnect wires are available on a large scale. Those LCRs replace the customary flat soldering ribbons for the series connection of solar cells. The idea behind this development is to increase the efficiency of a solar module by intelligent light redirecting while irradiating the ribbon's surface. The light is reflected back onto the surface of the cell via total internal reflection at the glass/air interface (Figs. 1 and 2). Up to $80 \%$ of the incoming light that strikes the ribbon is recovered compared to the $5 \%$ recovery of a traditional solar cell tabbing and interconnect wire. In combination with an electrical conductive adhesive (ECA) stringer, structured ribbons are easily processable. Concerning aesthetic rules in the building integrated PV, built modules with LCR technology appear very uniform and inconspicuous. A further advantage of the structure ribbon is the eco-friendly, lead-free process, fulfilling the directive on the restriction of hazardous substances (RoHS). In Figure 1 the typical process for redirecting light utilizing LCRs is illustrated. In step (1) light is reflected under a certain angle by the ribbon structure. The obliquely scattered light is totally reflected at the glass/air interface (2) and therefore reaches active cell material and generates charge carriers beside the ribbon (3).

The visual inspection shows the shiny surface of a flat ribbon associated with lossy light reflection (Figs. 3a, 3b right module). Compared to the flat ribbons, the LCRs appears darker, therefore less light is reflected and the embedded solar cell receives more light (Figs. 3a, 3b left module).

\subsection{Electrical conductive adhesive (ECA)}

For years, crystalline silicon (c-Si) cell - and module manufacturers have searched alternatives to replace the soldering - by a gluing process $[7,8]$. For this purpose, newly developed adhesives are present and especially suited for the variety of new cell concepts, such as

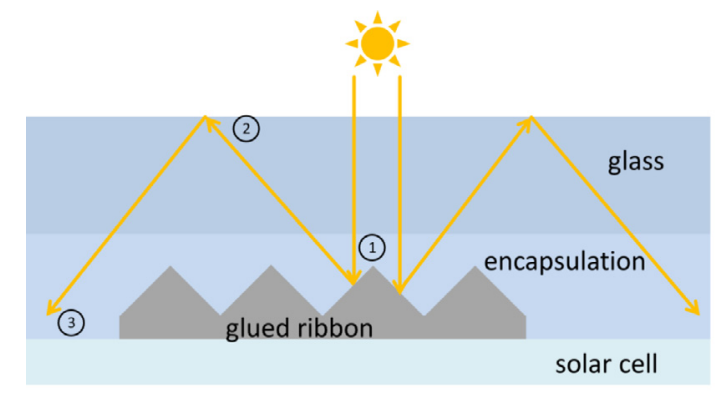

Fig. 1. Optical path of the light for a structured ribbon.

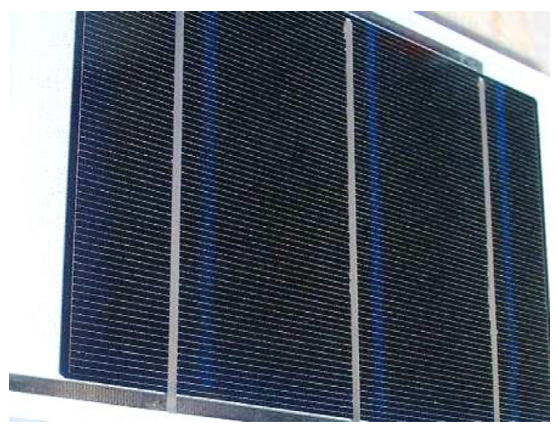

Fig. 2. Photograph of an irradiated mini module with emphasized LCR effect (blue lines beside LCRs).

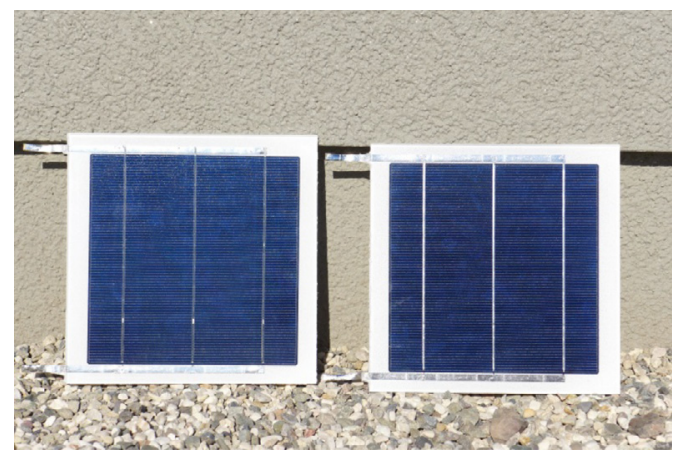

(a)

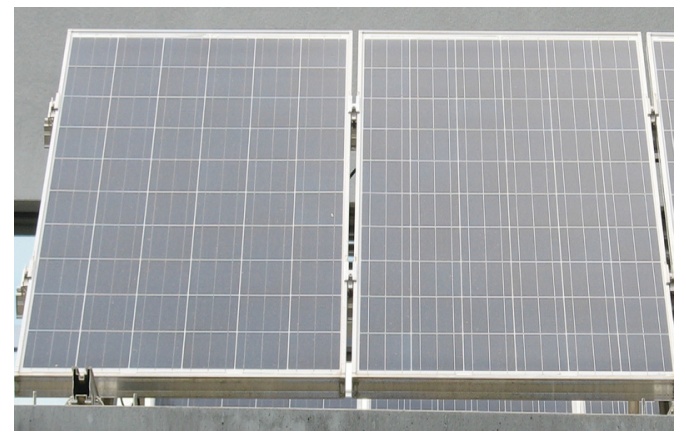

(b)

Fig. 3. (a) Photograph of an irradiated mini module with LCR ribbons (left) in comparison to a standard flat ribbon mini module (right). (b) Photograph of an irradiated module with LCR ribbons (left) in comparison to a standard flat ribbon module (right). 


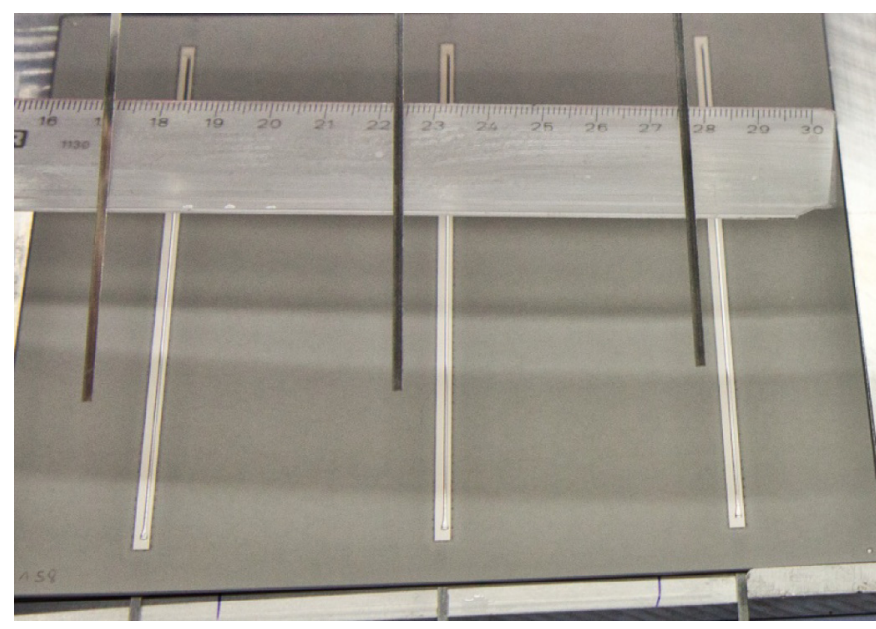

Fig. 4. Photograph of the gluing process with three busbars and ribbons.

back contact or $\mathrm{Cu}$-galvanic cells which allow cost reductions in many ways. The adhesive utilized in our tests (SoltaBond - SB 1242) is a standard electrical conductive, silver based adhesive, that can be used for c-Si cells in mass production (Fig. 4). In multiple test runs, the reliability of the epoxy resin adhesive on various surfaces has been proven. Due to the reduction in silver content, newest silver reduced glues are still in test phase but should minimize the overall adhesive costs. An important benefit of gluing is a decreased processing temperature of $180{ }^{\circ} \mathrm{C}$ compared to $220^{\circ} \mathrm{C}$ for soldering with the prospect of less stress in a solar cell, thus lowering the risk of tensions and micro cracks.

\section{Simulation}

The simulations are performed using a two-step algorithm programmed via MATLAB. The first step consists of a ray tracing algorithm. For a given cell geometry, determined by the cross-sectional shape of the ribbon and the thickness of the cover glass, the traces of 5000 parallel rays which are evenly distributed across the ribbon width are calculated. Trace calculation is aborted when a beam hits the plane of the solar cell, or, in order to avoid endless loops, if the number of impacts on the ribbon and the glass/air interface exceeds 200.

In the second step of the simulation, the effective intensity of each ray reaching the solar cell is calculated by forming the product of the transmission coefficient on the glass/air interface and the reflection coefficients of all subsequent reflections on the metal ribbon and the glass/air interfaces. Rays that do not reach the solar cell after 200 impacts are assigned with zero intensity. The dependence on the incident angle is taken into account for all interactions, except for the absorption in the cell, which is considered as a perfect absorber. As the incident light is not polarized, the average value of s- and p-polarized transmission and reflection coefficients is used. The average value of the effective intensity averaged over all rays

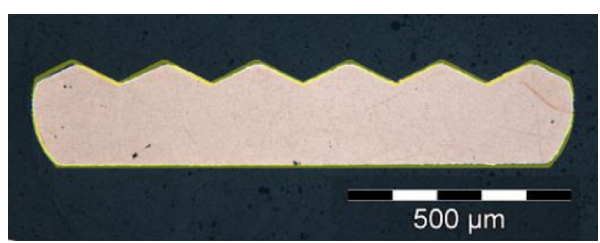

Fig. 5. Typical geometry of an LCR and its approximation for numerical simulations.

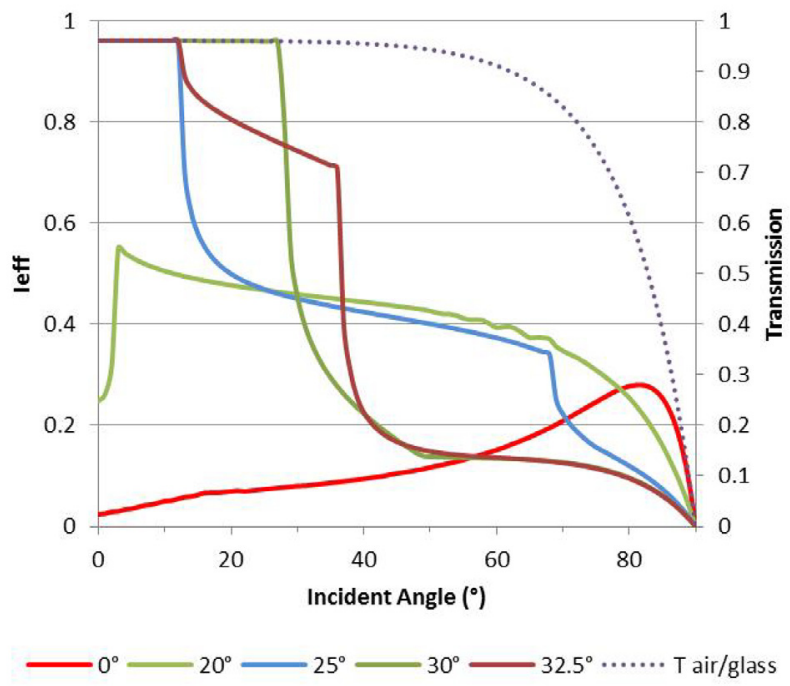

Fig. 6. $I_{\text {eff }}$ for different pyramid slope angles and corresponding incidence angles.

$\left(I_{\text {eff }}\right)$ describes the fraction of the light incident on the ribbon that is reflected back on the solar cell.

Having solved the purely geometrical problem of ray tracing in the first simulation step allows for a time efficient evaluation of $I_{\text {eff }}$ with respect to variations of the optical parameters involved in the problem. All simulations presented here were performed with a non-dispersive glass refractive index of 1.4, and a laminated glass/EVA thickness of $3.5 \mathrm{~mm}$. Figure 5 depicts the optical micrograph of a LCR cross section. The ribbon has an average height of $0.2 \mathrm{~mm}$ and $1.2 \mathrm{~mm}$ maximum width.

Referring to this geometry we investigated the efficiency dependence of LCRs on different geometrical parameters. In these calculations, the reflection coefficient at the metal surface was set equal to 1 for simplicity. The effective intensity was calculated for various values of the slope angle $\gamma$, and the incident angle as shown in Figure 6. Even for flat ribbons $\left(\gamma=0^{\circ}\right)$, a certain intensity reaches the cell. This is partly due to rays reflected from the ribbon's side boundary, but also by rays reflected back on the cell via the glass/air interface. Independent of the ribbon geometry, all curves approach zero for high incident angles due to the decreasing transmission of the air/glass interface. For a $20^{\circ}$ slope, the average intensity shows a pronounced increase around $3^{\circ}$ incident angle. The latter marks the onset where the rays, reflected from one side of the pyramids, are totally reflected at the glass/air interface. At higher slope angles both slopes contribute to light 


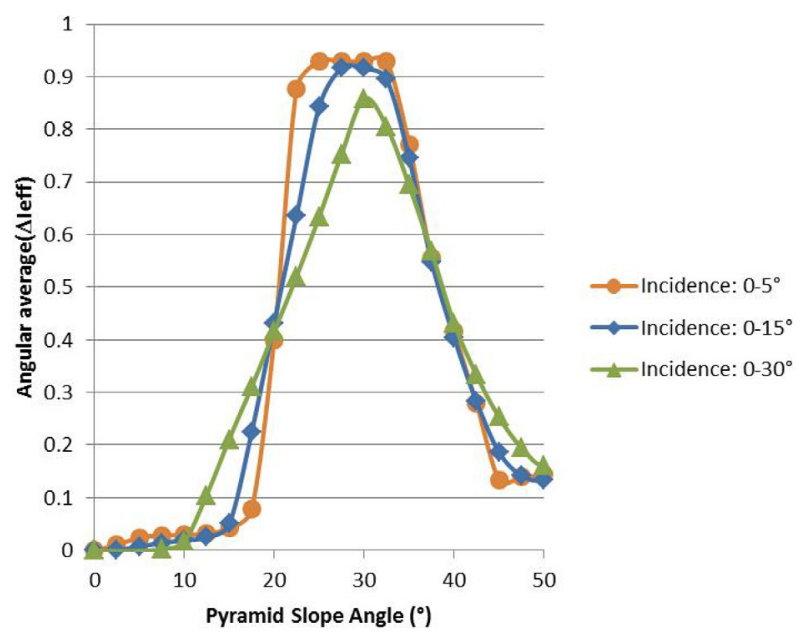

Fig. 7. Simulated different slope angles.

capturing via total reflection at small incident angles, and the range of the incident angle where this condition is fulfilled, broadens (see curves for slope angles of $25^{\circ}$ and $30^{\circ}$ in Fig. 6). At normal incidence, $I_{\text {eff }}$ reaches a value of 0.96 , which corresponds to the transmission of the air/glass interface and indicates that all incoming rays are redirected on the solar cell via total reflection. For higher slope angles $\left(32.5^{\circ}\right)$, multiple reflection on the LCR occurs which reduce the fraction of rays that are totally reflected. To verify the optimum value of the pyramid slope, we calculated the increase in effective average ray intensity:

$$
\Delta I_{\text {eff }}(\alpha)=I_{\text {eff }, \mathrm{LCR}}(\alpha)-I_{\text {eff,flat }}(\alpha)
$$

where $I_{\text {eff,LCR }}$ and $I_{\text {eff,flat }}$ are the effective average ray intensities of the light capturing ribbon and a flat ribbon, respectively, and $\alpha$ is the incident angle.

By averaging $\Delta I_{\text {eff }}(\alpha)$ over different ranges of incident angles around normal incidence, we obtain the dependencies shown in Figure 6. As long as only incident angles up to $15^{\circ}$ are taken into account, slope angles between $25^{\circ}$ and $32.5^{\circ}$ show the same increase. Considering a wider range of incidence from $0^{\circ}$ to $30^{\circ}$, however, it becomes obvious, that a slope angle around $30^{\circ}$ would be preferable in order to fully exploit the potential of the LCR, shown in Figure 7.

\section{Experimental set-up}

Eighteen single-cell mini module specimens were produced using the standard construction layers: glass (solar glass, low iron, $3.2 \mathrm{~mm}$ ), EVA (Vistasolar 486.10, $450 \mu \mathrm{m})$, pre-characterized solar cell (ETON, $220 \mu \mathrm{m}$ ), EVA (Vistasolar 486.10,450 $\mu \mathrm{m}$ ) and backsheet (ISOVOLTAIC APA $4004,350 \mu \mathrm{m})$. The solar cells are standard sized $(156 \mathrm{~mm}$ $\times 156 \mathrm{~mm}$ ) and consist of multi-crystalline silicon wafer material. Each cell is equipped with three busbars and connector dimensions of $152 \mathrm{~mm}$ lengths, $1.2 \mathrm{~mm}$ width and $0.2 \mathrm{~mm}$ depth. One third of the samples had standard soldered flat ribbons, one third had glued flat and one third glued structured ribbons. The copper ribbon core material has the same properties and dimensions for both, soldering and gluing ribbons. The only difference is the ribbon surface coating. The soldering ribbon uses a tin solder coating and the gluing ribbon uses a silver coating. The electrically conductive adhesive was a two components type (SB 1242). The string process was the same for soldered and glued cells. An infrared (IR) source connected the solar cell with the respective ribbons in the same machine. The soldering ribbons were soldered, the glued ribbons were cured on the busbars of the cell. Meanwhile the connection process every soldered as well as every glued ribbon was hold-down with 12 clamps at 12 points.

Initially we used single-cell mini modules to be more precise than with modules. The test criterion was the short circuit current (Isc) of the cell for a given amount of light. First, the specimens were measured under laboratory conditions, with illumination orthogonal to the cell, using a class AAA flash cell tester (Sinton FCT-350). Then, two sample pairs were measured again under direct sunlight and tilted to measure the effect of different illumination angles.

Upon making tests on single-cell mini modules, four 60-cells standard format modules were produced therefrom two equipped with the modified interconnector ribbons. Exactly the same way as for the single-cell mini modules, the 60-cells modules with soldered ribbons were compared to 60-cells modules with glued structured ribbons. The comparative Isc, $\mathrm{P}_{\mathrm{MPP}}$ flash cell test procedure was also repeated for the modules. Finally, the yield was determined by an outdoor test platform.

The results for the single-cell mini modules from the flash cell tester are described in Secion 5.1 and the angular dependent outdoor tests in Section 5.2.

The results for the flash module test for the standard 60 -cell modules with soldered and glued ribbons are described in Section 5.3 and the outdoor yield measurements in Section 5.4.

\section{Results}

\subsection{Single-cell mini modules measurements in the flash cell tester}

In a first step, a plurality of solar cells were measured and 18 cells sorted to equal Isc values. The selected cells were split into three groups, each with six cells for the ribbon investigations. Each cell was measured 5 times before and after encapsulation. The step from soldered flat ribbons with tin surface to glued flat ribbons with silver surface was taken into account too. Figure 8 shows the results including error bars. As expected, there is an average improvement from a six specimens set of glued structured ribbons to soldered ones of $2.03 \pm 0.46 \%$. The effect from soldered flat to glued flat ribbons is not relevant due to the margin of error bars. Therefore both string connection technologies, soldering or gluing, are possible without a significant difference in short circuit current (Fig. 8). 


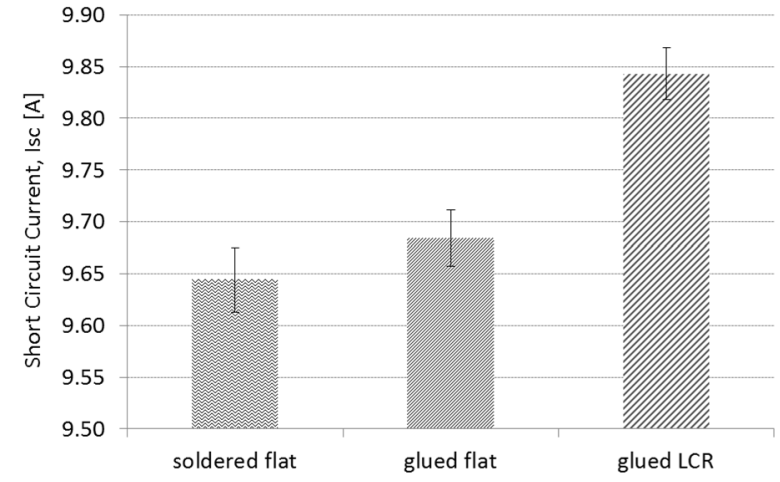

Fig. 8. Soldered standard ribbons compared to glued flat and glued LCRs in a cell flash tester.

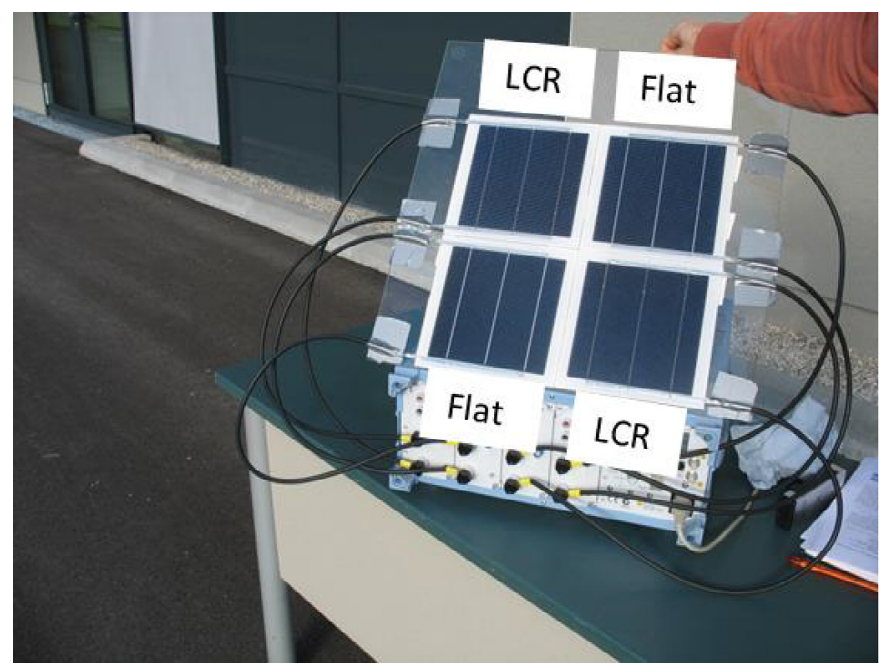

Fig. 9. Comparison of the different ribbons concerning short circuit current in tilted position.

\subsection{Single-cell mini modules outdoor measurements with angle dependence}

To characterize the angle dependence of structured ribbons, two mini modules with soldered and two with glued structured ribbons were mounted on a glass plate. With this arrangement two sample sets were measured simultaneously with the possibility to adjust any angle (Fig. 9). By measuring the Isc for the fixed samples, the quotient of the current for the structured ribbon sample to the standard soldered sample quantifies the benefit. The samples were oriented directly towards the sun using a rod. If the rod casts no shadow the sun is directly over the samples and the angle is defined to $0^{\circ}$. Two directions were tested vertical (Fig. 10) and horizontal (Fig. 11). In addition this experimental arrangement was verified by simulations.

The results of the blue sky outdoor measurements for the angle dependent case in vertical orientation is shown in Figure 10. Compared to the simulation curve with $5^{\circ}$ dispersion because of smoothed glass (black circle), the measurement points at different days (grey rhomb, black
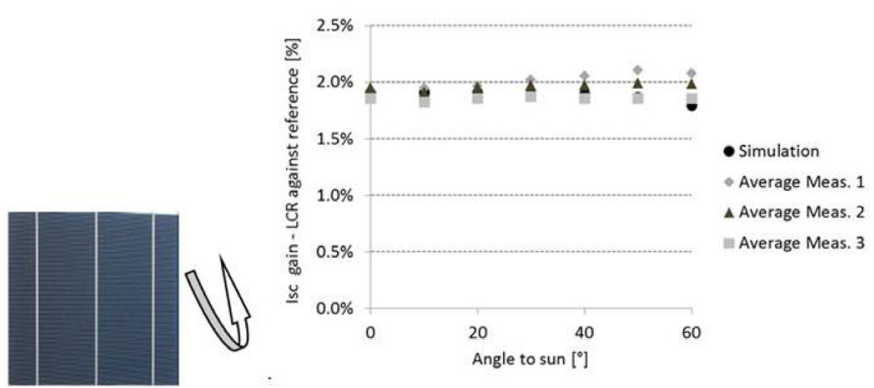

Fig. 10. Angle dependent gain of structured ribbons for vertical orientation of the ribbons.

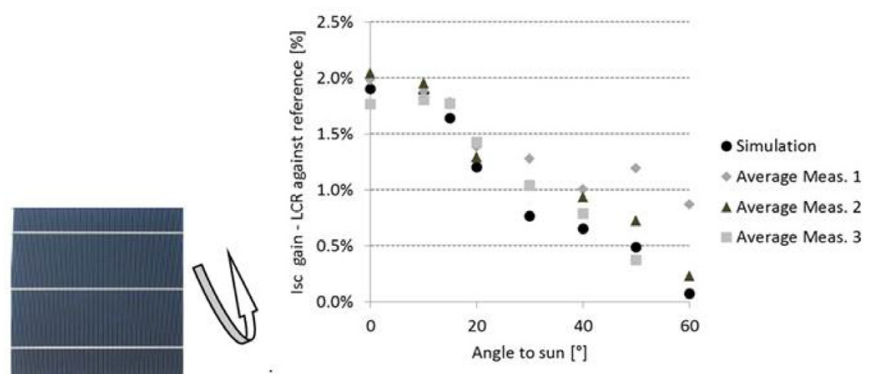

Fig. 11. Angle dependent gain of structured ribbons for horizontal orientation of the ribbons.

triangle, grey square) are in good agreement. Each measurement point is an average of 10 measurement points. While the simulations were done assuming a planar glass/air interface, the cover glass used for the fabrication of the modules had a corrugated surface. Thus, the angle of incident is not clearly defined. This variability was taken into account by averaging the simulations over a $5^{\circ}$ angular range, which corresponds to the surface topology of the glass surface.

The results of the outdoor measurements for the sample pair in horizontal orientation is shown in Figure 11. The measurement in comparison to the simulation is sectional in good agreement. For incident angles below $15^{\circ}$ all incoming light is reflected back on the solar cell. At higher angles, the condition of total internal reflection is no longer fulfilled for all rays and the gain decreases.

\subsection{0 -cells module measurements in the flash module tester}

Taking the step from single-cell mini modules to standard 60-cells modules is important to make sure that the structured ribbons work in a common module too. Hence four 60-cells modules with pre-sorted cells under variation in the ribbons type - soldered and glued LCR - were characterized using a calibrated flash module tester. Aware of the fact, that two modules per variation are not enough for a significant statistic, we emphasize that this test is a first indication whether the new technique and the module design are operating as expected. The relative gain in 


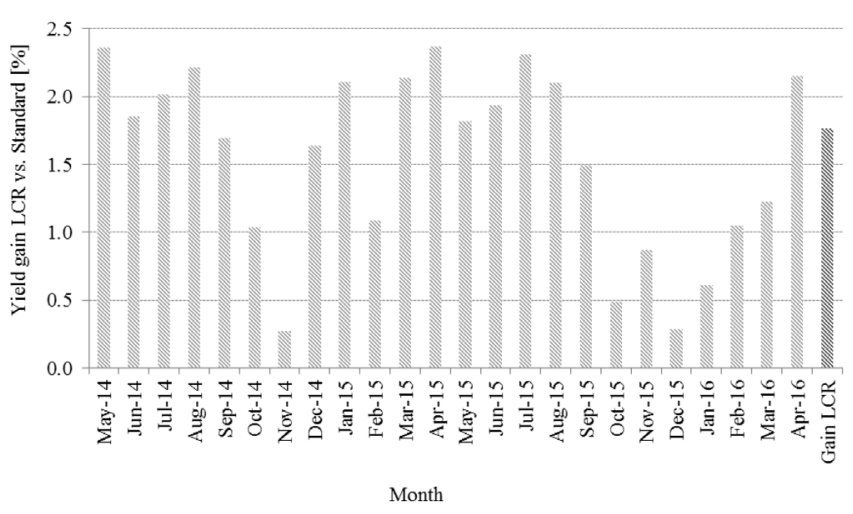

Fig. 12. Comparison of a module set over months - yield gain LCR against standard soldered.

Isc for module LCR 1 from Set 1 is $1.75 \%$ and for LCR 2 from Set 2 is $2.59 \%$ compared to the associated standard module. These values are in the range of plausibility. The gain in Isc for the single-cell mini modules is also affirmed for the 60-cells modules.

\subsection{0 -cells module measurements outdoor}

Not only the flash module test measurements provide relevant values for the yield of structured ribbons. In fact the yield in kilo watt hours $(\mathrm{kWh})$ over several days and weeks is of interest, if not the most interesting value for an end customer.

To test the effect on the yield using the new technique and ribbon design, the 60 -cells modules were mounted to a platform (Fig. 3b). The test platform was built on a shed and consists of two rows of modules which are optimally orientated to south direction under an angle of $26^{\circ}$. The climate, classified to category $\mathrm{C}$ in this south Austrian region, is moderate but warm with many rainfalls over the year.

Over a time period of two years the yield between a module set with glued LCR technology and a standard module with soldering ribbon technology (Set 1) is in the sun intensive months between 1.7 and $2.2 \%$. In the winter months the yield gain is jumpy and often around $1 \%$. So the LCR effect is more powerful in the summer. The $\mathrm{kWh}$ data per module were collected continuous with help of separate module boxes from Solaredge. In the end of every month the sum of collected $\mathrm{kWh}$ was inserted into a diagram. For the absolute yield gain for LCR vs. Standard ribbon technology, the ratio for all collected $\mathrm{kWh}$ in 24 months was relevant instead of an average of every month block. The gain in two years for LCR technology was $1.75 \%$ (Fig. 12).

\section{Discussion}

According to theory the reflectivity of a LCR ribbon is at $80 \%$. Calculated with the original dimensions for a three busbar solar cell, an expected Isc gain would be at $1.8 \%$. Compared to the measurements for the single-cell mini modules in a cell flash tester the Isc gain was at $2.03 \pm 0.46 \%$. Hence, expected and measured values were in good accordance.

The simulation and experimental comparisons for the angle dependence were in good agreement for vertical orientation. For the horizontal orientation the measurement showed a better behaviour than the simulation at angles from $30^{\circ}$ to $60^{\circ}$. This effect could be induced by the glass surface which was assumed to $5 \%$ roughness.

The yield measurements over 18 months resulted in a good accordance compared to the cell flash test results. The mean yield for test Set 1 LCR against standard flat ribbon was at $1.7 \%$ whereby the power gain from the cell flash test results was at $2.03 \pm 0.46 \%$. It is worth mentioning that the yield measurements are a combination of vertical and horizontal irradiation whereby the flash cell test results are only valid for one direction. Therefore it is plausible that the yield is somehow smaller than for Isc and Pmpp measurements in a cell flash tester, as the angle dependence investigations for vertical and horizontal orientations confirmed. Nevertheless, theory, simulation and experiments are in good agreement and show the potential of LCR technology.

\section{Conclusion}

In this project, conventional solar cells with soldered ribbons were compared to the novel design that uses glued structured ribbons (LCRs). First a mini module sample set of 18 equal cells was established. Each of the 18 cells was measured 5 times before and after lamination. The flash cell test results for the single-cell mini modules of precharacterized solar cells showed that the deeply structured glued ribbons have a higher short circuit current Isc (approx. $2.03 \pm 0.46 \%$ ) than soldered flat ribbons. Due to the fact that the spectrum of the flash cell tester differs from the solar spectrum, the experiment was repeated using natural light and almost identical results were observed. In general, light is not exactly orthogonal to the cell, but instead varies depending on time of day. Therefore, measurements on the performance of both, structured and flat connector types as a function of illumination angle were performed. When tilting a horizontal orientated ribbon sample in direct comparison to a flat ribbon sample, the LCR gain is at $1.8 \%$ for angles from $0^{\circ}$ to $15^{\circ}$ and drops to less than $1 \%$ at larger angles far away from $30 \%$. This is not true for the direct comparison in vertical tilting test. Over a wide range of angles the LCR gain is around $1.8 \%$ for angles from $0^{\circ}$ to $60^{\circ}$.

The same flash test procedure was conducted for the single-cell mini modules as well as for four 60-cells PV modules in a module tester. The flash test values for the power gain between glued LCR and soldered ribbons were around $1.5 \%$. This is in moderate accordance with the results for the measurements on single-cell mini modules described before. 
The attendant outdoor tests for measuring the yield over a longer time period confirmed the Isc gain for the LCR effect compared with a glued flat ribbon. The improvement in yield is at $1.7 \%$.

This work was conducted as part of the Austrian "Energy Research Program" project INFINITY, using results from the predecessor project InnoModu. Funded by the Austrian Climate and Energy Fund and the Austrian Research Promotion Agency (FFG), are both gratefully acknowledged.

\section{References}

1. L. Hamann, G.C. Glaeser, L. Proennecke, J.H. Werner, White Grid Fingers for Short Circuit Current Gain in Modules, in Proc. 26th EU PVSEC, Hamburg, Germany, 2011, pp. 487-491

2. A. Faes, M. Despeisse, J. Levrat, J. Champliaud, N. Badel, M. Kiaee, T. Söderström, Y. Yao, R. Grischke, M. Gragert, J. Ufheil, P. Papet, B. Strahm, B. Cattaneo, J. Cattin, Y. Baumgartner, A. Hessler-Wyser, C. Ballif, in Proc. 29th EU PVSEC, Amsterdam, The Netherlands, 2014, pp. 2555-2561
3. J. Schneider, M. Turek, M. Dyrbal, I. Baumann, B. Koll, T. Booz, Combined Effect of Light Harvesting Strings, AntiReflective Coating, Thin Glass and High UV Transmission Encapsulant to Reduce Optical Losses in Solar Modules, in Proc. 28th EU PVSEC, Paris, France, 2013, pp. 46-51

4. G.C. Gläser, L. Hamann, L. Prönneke, Structured Ribbons for Short Circuit Current Gain in Modules, in Proc. 25th EU PVSEC, Valencia, Spain, 2010, pp. 634-636

5. E.M. Sachs, J. Serdy, A.M. Gabor, F.V. Mierlo, LightCapturing Interconnect Wire for $2 \%$ Module Power Gain, in Proc. 24th EU PVSEC, Hamburg, Germany, 2009, pp. 3222-3225

6. Y. Zemen, M. Strasser, K. Turlapati, Performance and Yield Evaluation of c-Si Modules Built with Reflective Interconnectors, in Proc. 29th EU PVSEC, Amsterdam, The Netherlands, 2014, pp. 3302-3304

7. U. Eitner, T. Geipel, S.-N. Holtschke, M. Tranitz, Characterization of Electrically Conductive Adhesives, Energy Procedia 27, 676 (2012)

8. Md Zahidul Huq, Interconnection of front side busbar-free c-Si solar cells using conductive adhesives, Master thesis, University of Freiburg, Germany, 2013, https://www. researchgate.net/publication/261510083_Interconnection_ of_Front_Side_Busbar-Free_c-Si_Solar_Cells_Using_ Conductive_Adhesives_-_Md_Zahidul_Huq

Cite this article as: Wolfgang Muehleisen, Lukas Neumaier, Christina Hirschl, Thomas Maier, Michael Schwark, Siegfried Seufzer, Rene Battistutti, Mathias Pedevilla, Jög Scheurer, Robert Lorenz, Comparison of output power for solar cells with standard and structured ribbons, EPJ Photovoltaics 7, 70701 (2016). 